\title{
El desarrollo y movilización de competencias reflexivas en la formación de docentes de la Escuela Normal Oficial "Dora Madero"
}

\section{The development and mobilization of reflexive skills in teacher training of the Official Normal School "Dora Madero"}

\author{
GARCÍA-LÓPEZ, Francisco Enrique*†, HERNÁNDEZ-VALDEZ, María Magdalena, LUNA- \\ GARCÍA, Luis Alberto y FUENTES-FLORES, Moisés
}

Escuela Normal Oficial "Dora Madero”. Secretaría de Educación de Coahuila

ID 1 ${ }^{\text {er }}$ Autor: Francisco Enrique, García-López / ORC ID: 0000-0002-2970-8382

ID $1^{\text {er }}$ Coautor: María Magdalena, Hernández-Valdez / ORC ID: 0000-0002-5535-7301

ID $2^{\text {do }}$ Coautor: Luis Alberto, Luna-García / ORC ID: 0000-0002-0042-2209

ID $3^{\text {er }}$ Coautor: Moisés, Fuentes-Flores / ORC ID: 0000-0002-3391-2132

DOI: $10.35429 /$ JCP.2019.10.3.9.15

Recibido 16 de Agosto, 2019; Aceptado 30 de Diciembre, 2019

\section{Resumen}

El proceso de enseñanza aprendizaje implica un enfoque reflexivo del acto de enseñar. La reflexión es un componente esencial de todos los procesos inherentes a la formación de docentes. Es imprescindible fomentarla para potencializar el desarrollo de habilidades cognitivas y metacognitivas. Los actuales enfoques implican que los formadores de formadores transformen su práctica y los docentes en formación desarrollen diversas competencias para enfrentar los desafíos de la implementación de modelos educativos. El estudio es descriptivo, correlacional y factorial, además está basado en la investigación acción. La problemática parte de las diversas tensiones sobre el desarrollo de habilidades metacognitivas, (reflexión del aprendizaje. reflexión en y sobre la acción y la práctica reflexiva. En la formación de docentes, los procesos de reflexión se han ido fortaleciendo, por ello, este estudio se realizó para identificar los factores que facilitan el desarrollo de estas habilidades, las variables que están relaciones, el impacto de los procesos en el desarrollo de competencias y para conocer las acciones que realizan los profesores en los ambientes de aprendizaje. Actualmente el paradigma reflexivo ha cobrado un importante auge en las instituciones de educación superior porque se esta reconociendo la importancia del desarrollo de esta importante habilidad cognitiva.

Formación docente, Habilidades metacognitivas, Práctica reflexiva

\begin{abstract}
The teaching-learning process implies a reflexive approach to the act of teaching. Reflection is an essential component of all processes inherent in teacher training. It is essential to encourage it to potentiate the development of cognitive and metacognitive skills. Current approaches imply that trainers trainers transform their practice and teachers in training develop various skills to meet the challenges of implementing educational models. The study is descriptive, correlational and factorial, and is also based on action research. The problematic part of the various tensions about the development of metacognitive skills, (reflection of learning. Reflection in and about reflective action and practice. In teacher training, reflection processes have been strengthened, therefore, this study It was carried out to identify the factors that facilitate the development of these skills, the variables that are related, the impact of the processes in the development of competences and to know the actions that teachers perform in learning environments. It has gained a significant boom in higher education institutions because the importance of developing this important cognitive ability is being recognized.
\end{abstract}

Teacher training, Metacognitive skills, Reflective practice

Citación: GARCÍA-LÓPEZ, Francisco Enrique, HERNÁNDEZ-VALDEZ, María Magdalena, LUNA-GARCÍA, Luis Alberto y FUENTES-FLORES, Moisés. El desarrollo y movilización de competencias reflexivas en la formación de docentes de la Escuela Normal Oficial “Dora Madero”. Revista de Pedagogía Crítica. 2019, 3-10: 9-15

\footnotetext{
* Correspondencia al Autor (Correo electrónico: kikinkike@ hotmail.com)

$\dagger$ Investigador contribuyendo como primer autor.
} 


\section{Introducción}

La formación docente es un proceso complejo, entre otros aspectos, implica la planificación, implementación y la evaluación del proceso enseñanza aprendizaje; la realización de jornadas de práctica profesional; procesos sistemáticos de seguimiento, análisis, evaluación, reflexión, etc.

En los planes de estudio vigentes de la licenciatura en educación primaria (plan 2012 y 2018), el desarrollo de habilidades, entre ellas, las cognitivas son parte fundamental del modelo educativo para la formación inicial docente. Estas son herramientas indispensables para la construcción de significados y la atribución de sentido a los contenidos y experiencias por parte de la persona que aprende (Sep, 2012).

Es indiscutible que, en los estudiantes, mayormente, entre los que cursan los primeros semestres, se les dificultan los procesos de reflexión sobre el aprendizaje y otros aspectos inherentes a su formación. En el resto, particularmente de los semestres del 6 al 8vo, estas habilidades se han desarrollado en mayor medida, sin embargo, aún se siguen presentando oportunidades de mejora, las cuales son evidentes al momento de que estos realizan ejercicios de análisis y reflexión sobre su propio aprendizaje y/o de las jornadas de práctica profesional.

En la acción docente, la reflexión es sustancial en el acompañamiento académico para incidir en la adquisición de diversos saberes. La reflexión está directamente relacionada con los procesos cognitivos y socioafectivos, porque implica desarrollar cierto conjunto de habilidades intelectuales y asumir de manera crítica el proceso de reflexión, lo que implica regular las emociones y sentimientos. Desde la perspectiva de diversos autores, la reflexión contribuye en la metacognición, la autoeficacia académica, la autorregulación del aprendizaje y en el cambio de actitud al regular de manera crítica lo socio emocional.

Para el estudio se plantearon interrogantes, hipótesis y objetivos.

\section{Preguntas de investigación}

¿Qué factores están relacionados con el desarrollo de la reflexión?
¿Cuáles son los efectos de los procesos metacognitivos en el aprendizaje y desempeño de los estudiantes durante las jornadas de práctica profesional?

\section{Hipótesis}

El desarrollo de la actitud reflexiva modela la forma durable de la capacidad de aprender, de reflexionar sobre la acción y de transformarla.

\section{Objetivos de investigación}

- Explorar los procesos de metacognición que se implementan en los ambientes de aprendizaje de la escuela normal.

- Conocer las relaciones entre los procesos y estrategias que se desarrollan en las aulas de clase.

- $\quad$ Identificar las principales dimensiones y factores relacionados con el desarrollo de competencias reflexivas en la formación de docentes.

\section{Metodología a desarrollar}

Se aplicó el enfoque cuantitativo para explorar el fenómeno, elaborar descripciones generales e identificar relaciones entre las variables. $\mathrm{La}$ investigación está en sus primeras etapas. La muestra para el estudio cuantitativo fue de 68 escolares del ciclo 2018-2019 del 2do, 4to, 6to y 8 vo semestres. La recogida de datos fue a través de encuestas tipo Likert. El objeto de estudio, en este caso, lo son el desarrollo de habilidades de reflexión, la reflexión sobre el aprendizaje y la reflexión sobre las prácticas profesionales.

La encuesta tipo Likert se conformó con 17 variables relacionadas con la formación de estudiantes y practicantes reflexivos basado en los postulados de Perrrenoud (2004), Cerecero (2016) además de diversas propuestas teóricas y metodológicas, entre ellas las de Valladares (2011), Scallon, 2000, citado en Fernández, 2010, Gutiérrez, (s.f.), entre otros.

\section{Resultados}

Los datos recopilados obtuvieron un $88 \%$ de confiabilidad y un nivel de error del 12\% (Alpha de Cronbach). El estudio exploratorio permitió conocer la percepción que tienen los estudiantes sobre el fenómeno de estudio.

GARCÍA-LÓPEZ, Francisco Enrique, HERNÁNDEZ-VALDEZ, María Magdalena, LUNA-GARCÍA, Luis Alberto y FUENTES-FLORES, Moisés. El desarrollo y movilización de competencias reflexivas en la formación de docentes de la Escuela Normal Oficial "Dora Madero". Revista de Pedagogía Crítica. 2019 
Estos manifiestan que en los ambientes de aprendizaje de la escuela normal, los procesos de reflexión están contribuyendo a formar a un profesional capaz de dominar su propia evolución, construyendo competencias y saberes nuevos o más precisos a partir de lo que ha adquirido y de la experiencia $(\mathrm{x}=4.3)$, producto de la formación continua para el desarrollo del saber analizar y de la actitud reflexiva $(x=4.3)$, aunado a que en el diseño del plan de formación, este está siendo organizado alrededor de las competencias $(\mathrm{x}=4.2)$. Los resultados de estas variables permiten identificar las variables relacionadas con el desarrollo de la reflexión.

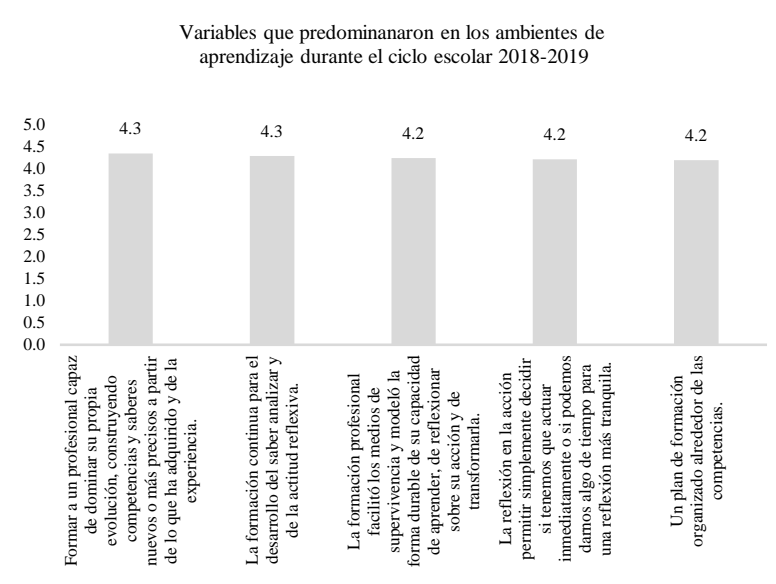

Gráfico 1 Variables que predominaron en los ambientes de aprendizaje

Desde la perspectiva de los sujetos de estudio, estos plantean que en el proceso de enseñanza aprendizaje se están fomentando: La reflexión en la acción, lo que provoca a menudo una reflexión sobre la acción, porque pone «en reserva» cuestiones imposibles de tratar en el momento, pero que el estudiante/practicante quiere volver a analizar «con más calma»; los educandos consideran que el desarrollar la actitud reflexiva está facilitando la adquisición de conocimientos y el saber- hacer correspondientes; los primeros resultados del estudio señalan que la formación que están recibiendo los estudiantes está modelando de forma durable su capacidad para aprender $(\mathrm{x}=4.2)$ además, de reflexionar sobre su acción y de transformarla.

Esta triada de indicadores obtuvo una media de $x=4.2$. estos datos evidencian que los estudiantes están desarrollando la capacidad de reflexionar sobre el sistema y las estructuras de la acción individual o colectiva.
La actividad reflexiva no es ajena a los procesos de planeación ni de evaluación, sino que está estrechamente vinculada con la planeación didáctica y la evaluación autentica, es decir, a la evaluación formativa del aprendizaje y de las competencias, en donde la retroalimentación por parte del docente es un elemento fundamental e imprescindible, para contribuir en el desarrollo de habilidades metacognitivas. Ver Tabla 1 ¿En qué se centran los ambientes de aprendizaje?

\section{Variables}

\begin{tabular}{|l|l|l|}
\hline Formar a un profesional capaz de dominar su propia & 4.3 & 0.52
\end{tabular}

evolución, construyendo competencias y saberes nuevos o más precisos a partir de lo que ha adquirido y de la experiencia.

\begin{tabular}{|l|l|l|}
\hline La formación continua para el desarrollo del saber & 4.3 & 0.29
\end{tabular} analizar y de la actitud reflexiva.

\begin{tabular}{|l|l|l|l|}
\hline La formación profesional facilitó los medios de & 4.2 & 0.29
\end{tabular} supervivencia y modeló la forma durable de su capacidad de aprender, de reflexionar sobre su acción y de transformarla.

\begin{tabular}{|ll|l|l|}
\hline La reflexión en la acción permitir simplemente & 4.2 & 0.29
\end{tabular} decidir si tenemos que actuar inmediatamente o si podemos darnos algo de tiempo para una reflexión más tranquila.

\begin{tabular}{|l|l|l|l|}
\hline Un plan de formación organizado alrededor de las & 4.2 & 0.51 \\
\hline
\end{tabular} competencias.

\begin{tabular}{|lllll|l|l|}
\hline Desarrollar la actitud reflexiva y facilitar los & 4.1 & 0.51
\end{tabular} conocimientos y el saber- hacer correspondientes

\begin{tabular}{|l|l|l|l|}
\hline Desarrollar la capacidad de reflexionar sobre el & 4.1 & 0.62
\end{tabular} sistema y las estructuras de la acción individual o colectiva.

La reflexión en la acción

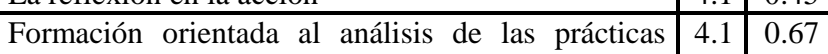
profesionales y a los procedimientos de formación docente.

\begin{tabular}{|l|l|l|}
\hline La evaluación formativa de las competencias. & 4.0 & 0.67 \\
\hline
\end{tabular}

\begin{tabular}{|l|l|l|l|}
\hline Desarrollar la capacidad de reflexionar sobre la & 4.0 & 0.72
\end{tabular}

acción en el recorrido previo y posterior de los momentos de compromiso intenso con una tarea o una interacción.

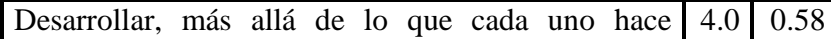
espontáneamente, la capacidad de reflexionar en plena acción.

\begin{tabular}{|l|l|l|l|}
\hline Una transposición didáctica fundada en el análisis de & 4.0 & 0.51 \\
\hline
\end{tabular} la práctica y de sus transformaciones.

\begin{tabular}{|l|l|l|}
\hline Multiplicar las ocasiones para que los estudiantes en & 4.0 & 0.83
\end{tabular}

las aulas y en prácticas se forjen esquemas generales

de reflexión.

\begin{tabular}{|l|l|l|}
\hline Una verdadera articulación entre teoría y práctica. & 4.0 & 0.49 \\
\hline
\end{tabular}

\begin{tabular}{|l|l|l|}
\hline Una reflexión regular y precisa sobre la mayor parte & 3.9 & 0.49 \\
\hline
\end{tabular}

de las acciones singulares, en curso, pasadas o previstas.

\begin{tabular}{|llll|l|l|}
\hline Un entrenamiento cognitivo más intensivo y & 3.8 & 0.51 \\
\hline
\end{tabular} controlado

Tabla 2 ¿En qué se centran los ambientes de aprendizaje?

La evaluación formativa de las competencias $(\mathrm{X}=4.0)$ en la escuela normal ha mejorado, en comparación con otros ciclos escolares, lo que está favoreciendo una mejor medición del desarrollo de estas.

GARCÍA-LÓPEZ, Francisco Enrique, HERNÁNDEZ-VALDEZ, María Magdalena, LUNA-GARCÍA, Luis Alberto y FUENTES-FLORES, Moisés. El desarrollo y movilización de competencias reflexivas en la formación de docentes de la Escuela Normal Oficial "Dora Madero". Revista de Pedagogía Crítica. 2019 
Para los estudiantes la reflexión en la acción les está permitiendo decidir si tienen que actuar inmediatamente o si pueden darse algo de tiempo para una reflexión más tranquila, aunado al desarrollo de la capacidad de reflexionar sobre la acción, en el recorrido previo y posterior de los momentos de compromiso intenso con una tarea o una interacción. Por ello es importante desarrollar, más allá de lo que cada uno hace espontáneamente, la capacidad de reflexionar en plena acción.

Este primer acercamiento al fenómeno de estudio (exploratorio/descriptivo) ha permitido reconocer lo que está pasando en el desarrollo de habilidades cognitivas y metacognitivas. Es evidente que los procesos de reflexión están aportando importantes beneficios. Los resultados demuestran que durante la formación inicial de docentes se está haciendo énfasis en: un entrenamiento cognitivo más intensivo y controlado; una formación más orientada al análisis de las prácticas profesionales y a los procedimientos de formación docente; el fortalecimiento de la articulación entre teoría y práctica; una reflexión más regular y precisa sobre la mayor parte de las acciones singulares, en curso, pasadas o previstas; multiplicando las ocasiones para que los estudiantes en las aulas y en prácticas se forjen esquemas generales de reflexión; y una transposición didáctica fundada en el análisis de la práctica y de sus transformaciones.

\section{El desarrollo de habilidades metacognitivas en la formación de docentes}

Para conocer posibles relaciones entre las variables del instrumento, se realizó un estudio correlacional, ya que este permite identificar como se asocian variables mediante un patrón predecible para un grupo o población. Este tipo de estudios tiene como finalidad conocer la relación o grado de asociación que exista entre dos o más conceptos, categorías o variables en una muestra o contexto en particular. (Hernández, 2016). Las variables Un plan de formación organizado alrededor de las competencias y La formación continua para el desarrollo del saber analizar y de la actitud reflexiva obtuvieron un grado de vinculación o correlación significativa de $r=0.619$ en el nivel 0,01 (bilateral), las variables
La formación profesional facilitó los medios de supervivencia y modeló la forma durable de su capacidad de aprender, de reflexionar sobre su acción y de transformarla y Una reflexión regular y precisa sobre la mayor parte de las acciones singulares, en curso, pasadas o previstas fue de $\mathrm{r}=0.562$, otras correlaciones significativas bilaterales los fueron La evaluación formativa de las competencias y Un entrenamiento cognitivo más intensivo y controlado con $\mathrm{r}=0.545$; Multiplicar las ocasiones para que los estudiantes en las aulas y en prácticas se forjen esquemas generales de reflexión y Formación orientada al análisis de las prácticas profesionales y a los procedimientos de formación docente con una correlación de $\mathrm{r}: 0.523$.

Los anteriores coeficientes de correlación son importantes porque ayudan a explicar lo que está sucediendo en la formación de docentes.

\section{La evaluación formativa de las competencias y los esquemas generales de reflexión}

En la tabla 2, se observa que resultaron dos factores, que subyacen del método de extracción Análisis de componentes principales, de tal modo que se relacionaron 17 variables, para obtener los atributos de la propuesta, con un nivel de explicación del $44.58 \%$. En cuanto a la formación docente a través de la planeación y evaluación por competencias para el desarrollo de capacidades reflexivas.

\begin{tabular}{|l|l|l|l|}
\hline $\begin{array}{c}\text { Eigen } \\
\text { Value }\end{array}$ & $\begin{array}{c}\text { \% Total de la } \\
\text { Varianza }\end{array}$ & \multicolumn{1}{|c|}{$\begin{array}{c}\text { Valores Propios } \\
\text { Acumulados }\end{array}$} & $\begin{array}{c}\text { Acumul } \\
\text { ado\% }\end{array}$ \\
\hline 5.908 & 34.754 & 5.908 & 34.754 \\
\hline 1.671 & 44.584 & 1.671 & 44.584 \\
\hline
\end{tabular}

Tabla 2

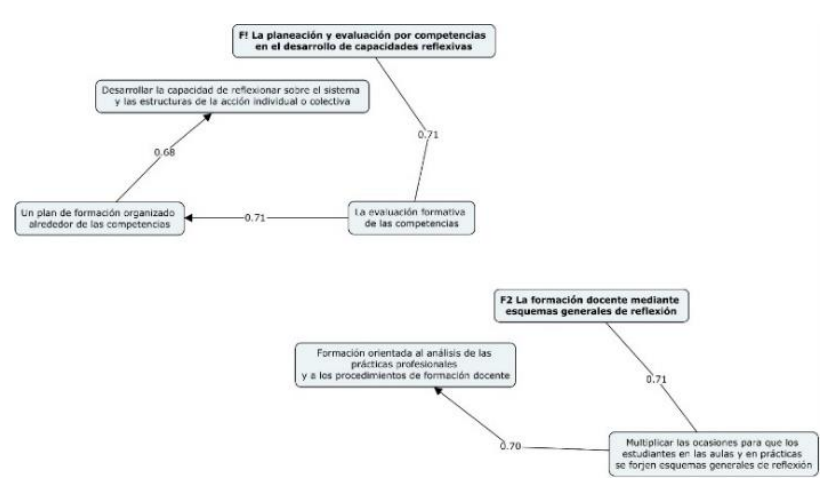

Figura 1 La relación interfactorial entre la planeación y evaluación por competencias en el desarrollo de capacidades reflexivas y la formación docente mediante esquemas generales de reflexión 


\section{Lectura Interfactorial}

La planeación y evaluación por competencias en el desarrollo de capacidades reflexivas (F1) a partir de la formación docente mediante esquemas generales de reflexión (F2)

\section{Análisis Integracional Factor 1}

En la gráfica anterior se observa que la evaluación formativa de las competencias aunado al diseño de un plan basado en competencias está favoreciendo en los estudiantes el desarrollo de la capacidad reflexiva sobre el sistema y las estructuras de la acción tanto en el ámbito individual como colectivo. Perrenoud (2004) plantea que este tipo de reflexión sobre las estructuras de la acción tiene sus raíces, en general, en una reflexión regular y precisa sobre la mayor parte de las acciones singulares, en curso, pasadas $\mathrm{o}$ previstas.

Este mismo autor plantea que en un primer nivel, la reflexión sobre nuestro sistema de acción cuestiona los fundamentos racionales de la acción: las informaciones disponibles, su tratamiento, los conocimientos y los métodos con los que nos ayudamos.

\section{Análisis Integracional Factor 2}

En el segundo factor se muestra que la formación docente se está realizando mediante esquemas generales de reflexión como consecuencia de las múltiples ocasiones en que los estudiantes en las aulas y en las prácticas profesionales la están fomentando. Dicha formación orientada al análisis de las prácticas profesionales y a los procedimientos de formación docente se sustentan en los postulados de la práctica reflexiva, la cual se aprende con un entrenamiento intensivo, característica primordial de la reflexividad.

\section{Conclusiones}

Los primeros hallazgos del estudio han permitido identificar factores que están relacionados con el desarrollo de la reflexión y conocer cuáles son los efectos de los procesos metacognitivos en el aprendizaje y desempeño de los estudiantes durante las jornadas de práctica profesional, particularmente desde la perspectiva los estudiantes.
Al principio se advirtió que los estudiantes de octavo semestre no tenían mucha disposición, fue la primera impresión, para responder los cuestionarios, dialogar y realizar los análisis de la jornada de práctica profesional, en comparación con los estudiantes de recién ingreso.

Este fenómeno también se identificó en menor medida entre los alumnos de quinto semestre. Entre los principales argumentos, destacaron que tenían demasiado trabajo de planeación, diseño de formatos y recursos, revisión de tareas, elaboración del diario de campo/trabajo, evaluación de actividades, etc.

Es preocupante, porque en la medida de que el estudiante avanza en su formación, se incrementan los alcances de las jornadas de práctica profesional y aumentan las cargas curriculares y cocurricualres, por lo que en la práctica se percibe que están disminuyendo la frecuencia y número de horas que dedican a los procesos de reflexión.

Estas condiciones reales de trabajo docente están teniendo un impacto negativo en la profundidad y calidad de los análisis, en el desarrollo o consolidación de competencias genéricas y profesionales y por consecuencia están propiciando la generación de nuevas tensiones en la formación de docentes, particularmente en la última etapa de formación.

En esta primera etapa del estudio se plantearon tres objetivos; Explorar los procesos de metacognición que se implementan en los ambientes de aprendizaje de la escuela normal, Conocer las relaciones entre los procesos y estrategias que se desarrollan en las aulas de clase e Identificar las principales dimensiones y factores relacionados con el desarrollo de competencias reflexivas en la formación de docentes, para ello, los datos recabados fueron analizados mediante estadística descriptiva, correlación y análisis factorial.

Los resultados son importantes indicios de lo que esta sucediendo en los ambientes de aprendizaje y lo que esta propiciando el desarrollo de habilidades cognitivas, particularmente la reflexión, lo cual no es un proceso aislado, sino que implica la movilización de diversas capacidades. 
Producto del análisis de los resultados de la reflexión sobre el aprendizaje, del desarrollo de competencias y de las jornadas de prácticas profesionales, los sujetos de estudio afirman que al fomentarse la reflexión en la formación de docentes y en el proceso de aprendizaje, se facilita la comprensión y análisis de la información, dichos procesos crean un aprendizaje de calidad; les permite ver las situaciones que se les presentan desde otra perspectiva; pueden identificar lo que están haciendo bien y lo que no, además de que facilita la autoevaluación, la comprensión de textos, la mejora de la redacción de textos; escribir de manera correcta y con orden las ideas que quieren expresar; aprender a analizar las lecturas.

Esta etapa de la investigación permitió identificar cómo la reflexión sistemática sobre el proceso de enseñanza aprendizaje ha impactado en la formación profesional, entre lo que se destaca es que está favoreciendo el desarrollo del pensamiento crítico, mejoras en la redacción y a complementar las actividades de aprendizaje con las reflexiones que están realizando. Respecto a ¿Qué factores están relacionados con el desarrollo de la reflexión?, los resultados muestran que la planeación y la evaluación por competencias están favoreciendo el desarrollo de capacidades reflexivas, lo que está permitiendo entre los estudiantes reflexionar sobre la acción individual y colectiva.

Otro de los cuestionamientos de esa investigación lo fue ¿Cuáles son los efectos de los procesos metacognitivos en el aprendizaje y desempeño de los estudiantes?, se resalta que los educandos están formándose esquemas generales de reflexión, producto de la formación orientada al análisis de las prácticas profesionales y a los procedimientos de formación docente. Los resultados demuestran que cuando los estudiantes reflexionan sobre lo que han conseguido aprender, les permite adquirir más conocimientos con mayor facilidad, investigar en más fuentes y seleccionar mejor la información, adicionalmente, consideran que tienen los insumos necesarios para realizar buenas prácticas (diseño de actividades y elaboración de instrumentos de evaluación), y que la autorreflexión de lo que hacen es esencial, porque en comparación cuando esta no la realizaban, lo hacían sólo por cumplir, lo que generaba el estancamiento en el desarrollo de sus habilidades.
Otros resultados destacan que como producto de la reflexión han conseguido ser autocríticos en su propio actuar tanto en la escuela normal como en las escuelas de práctica. Fomentar la reflexión está impactando en el desarrollo de otras habilidades metacognitivas, lo que les está permitiendo a los docentes en formación ser autocríticos, reconocer sus logros, identificar sus oportunidades de mejora y asumir con mayor responsabilidad y compromiso los retos y desafíos que la docencia demanda. Reflexionar sobre la práctica profesional, es otro de las categorías de estudio.

Para ello se diseñaron diversos cuestionarios y un diario de campo, con propuestas metodológicas afines. Estas primeras etapas de la investigación también permitieron identificar que aún persisten tensiones entre los docentes normalistas sobre el desarrollo de diversas habilidades, entre ellas, la reflexión, no sólo de aprendizaje, sino también de las prácticas que ellos mismos realizan y las que los estudiantes desarrollan como parte de su formación como docentes.

Se han multiplicado las acciones para fomentar los procesos de reflexión, lo que evidentemente está contribuyendo al desarrollo de una cultura del habitus de reflexión. Determinadas teorías señalan que durante el desarrollo de esta habilidad se pueden generar un sin de tensiones, entre ellas, la inefectividad de las interacciones en el aula, diferencias entre los docentes, debido a las desiguales perspectivas que tienen los docentes en los cuerpos colegiados acerca de cómo desarrollar estas habilidades, de si son o no valiosas para la formación profesional, la frecuencia y cantidad de las acciones durante el acompañamiento, etc. La reflexión debe desarrollarse de manera gradual y ser cuidadosamente planificada.

Los formadores y docentes en formación tienen que asegurarse de que sus acciones sean congruentes con el discurso, de tal manera que los juicios críticos que realicen no se desestimen con la implementación de acciones impulsivas, faltas de reflexión, carentes de un constante análisis y diálogo, no sólo consigo mismo, sino también con la comunidad académica, distantes de la reflexión y la racionalidad crítica y autocrítica. Tal como lo señala Perrenoud (2004) las universidades están pobladas de eruditos que no saben enseñar y que no se plantean nada sobre el tema. 
Cuando se han desarrollado estas habilidades, estaremos sistemáticamente fomentando el Autoconocimiento (proceso de aprendizaje y en las actitudes); accediendo a planos superiores de valoración crítica; reflexionando acerca de los procesos de aprendizaje (metacognición); fomentando la racionalidad autocrítica (Edgar Morin, 2006, citado en Pimienta, 2012.)

\section{Referencias}

Braun, A. (Febrero de 2006). Universidad de Palermo. Obtenido de http://fido.palermo.edu/servicios_dyc/publicaci onesdc/vista/detalle_articulo.php?id_articulo $=8$ 25\&id_libro=122

Cerecero, I. (2018). Práctica reflexiva mediada. Toluca: Editorial Académica Española.

Pimienta, J. (2012). Las competencias en la docencia universitaria. México: Pearson Educación de México, S.A. de C.V.

Redfforma - OEI - CRIFPE. (2018). CONGRESO INTERNACIONAL SOBRE LA FORMACIÓN Y LA PROFESIÓN DOCENTE. Santiago, Chile: Redfforma - OEI - CRIFPE.

Schettini, P., \& Cortazzo, I. (2015). Análisis de datos cualitativos en la investigación social. Procedimientos $y$ herramientas para la interpretación de información cualitativa. La Plata: Universidad de la Plata.

Shulman, L. (2005). CONOCIMIENTO Y ENSEÑANZA: FUNDAMENTOS DE LA NUEVA REFORMA. Revista de currículum y formación del profesorado, 1-30.

Valladares, M. (2011). Centro Tecnológico Ucampus de la Universidad de Chile. Obtenido de https://www.u-cursos.cl/ 